\title{
ORT_07 - Use of mammary tumor spheroids to study in vitro metastatic potential and therapeutic response
}

Laura Lacerda Coelho ${ }^{1}$; Matheus Menezes Vianna ${ }^{1}$; Debora Moraes da Silva ${ }^{1 *}$; Ana Carolina Monteiro ${ }^{1}$; Adriana Bonomo ${ }^{1}$; Pedro Paulo de Abreu Manso'; Fernando Regla Vargas ${ }^{1}$; Luciana Ribeiro Garzoni ${ }^{1}$. ${ }^{1}$ Fiocruz/IOC.

Introduction: Breast cancer is the most prevalent cancer among women worldwide and in Brazil. Although, great scientific advances have provided therapeutic innovations, it is still a challenge therapeutic agents that act against metastasis. The three-dimensional (3D) systems of cell culture better mimic the molecular, morphological and functional features of in vivo tumor than traditional monolayer cultures (2D), working as an ideal platform for understanding cancer biology and to perform therapeutic response analysis.

Objective: The aim of this study was to establish a scaffold-free 3D spheroid model to investigate in vitro metastatic potential and therapeutic response of tumor cells during doxorubicin treatment.

Methodology: First, we established and characterized the 3D model by analyzing PI, Ki-67, E-cadherin and laminin staining in spheroids of MCF-7. Then, we produced spheroid from cells isolated from mouse mammary tumors produced from $4 \mathrm{~T} 1$ and $67 \mathrm{NR}$ cell lines. We also characterized the migration/metastasis assay by evaluating E-cadherin and Vimentin expression (epithelial-mesenchymal transition markers) and cellular dispersion of MCF-7 cells. Even, we validated the model for in vitro therapeutic response studies using doxorubicin (dox), evaluating spheroid diameter, cell death, cell viability and migration/metastatic potential of cells.

Results: Mammary tumor spheroids (MTS) produced from the MCF-7 cell line mimicked avascular tumor characteristics, such as necrotic center and peripheral proliferative cells. In addition, MTS exhibited adherent junction proteins and were able to produce their own extracellular matrix. We also demonstrate that the spheroid model supports the 3D culturing of cells isolated from mouse mammary tumors produced from 4T1 and 67NR cell lines. Through the cell migration assay, we demonstrated for the first time that this model reproduces the epithelial-mesenchymal transition process (EMT), characteristic as one of the steps of metastasis formation and verified by the loss of E-cadherin protein and increased vimentin expression, as cells move away from "tumor" in vitro. We also observed collective cell migration, another feature of the metastatic process. Dox induced cell death, reduced spheroid diameter and inhibited the metastatic potential of tumor cells in vitro, by reducing collective migration and inhibiting the EMT process, suggesting a new application for this drug in anti-cancer treatment.

Conclusion: Our results demonstrate that $3 \mathrm{D}$ cultivation reproduced characteristics of breast tumors observed in vivo, including the metastatic process sensitive to dox treatment, suggesting that mammary tumor spheroids may be considered a powerful in vitro tool for the study of metastatic potential of tumor cells and new therapeutic approaches against breast cancer.

Keywords: Breast cancer; spheroids; epithelial-mesenchymal transition 